\title{
REDUCCIÓN DE LA PERMEABILIDAD CORNEAL EN PACIENTES TRATADOS CON HP-GUAR: ESTUDIO FLUOROFOTOMÉTRICO
}

\author{
REDUCTION OF CORNEAL PERMEABILITY IN PATIENTS \\ TREATED WITH HP-GUAR: A FLUOROPHOTOMETRIC STUDY
}

\author{
CERVÁN-LÓPEZ I I ${ }^{1}$, SÁENZ-FRANCÉS-SAN-BALDOMERO F ${ }^{1}$, BENÍTEZ-DEL-CASTILLO JM² \\ GARCÍA-SÁNCHEZ J²
}

\begin{abstract}
RESUMEN
Introducción: El ojo seco es una enfermedad en la que entre otras estructuras se afecta el epitelio corneal. El tratamiento de primera línea siguen siendo los sustitutivos lagrimales que buscan lograr una buena humidificación y lubricación del epitelio corneal de estos pacientes, evitando así su progresivo deterioro funcional. El HP-Guar es un compuesto novedoso cuyos resultados sintomáticos en los pacientes con ojo seco están siendo prometedores. Métodos: Presentamos un estudio prospectivo, enmascarado, pareado sobre 10 ojos izquierdos de 10 pacientes con ojo seco, a los que se les realizó medidas de la permeabilidad corneal mediante fluorofotometría con la instilación de 40 microlitros de una solución de fluoresceína sódica al 2\%, antes y después del tratamiento con HP-Guar. Se analizaron los resultados con el test de Wilcoxon para datos pareados.
\end{abstract}

\begin{abstract}
Introduction: The incidence of dry eye disease, which mainly affects the corneal epithelium, is rising. The main treatment is still the use of artificial tears capable of improving the humidification and lubrication of the corneal epithelium, and avoiding its progressive functional failure. HP-Guar is a new compound used for this purpose.

Methods: We performed a prospective, masked and paired study on the left eye of 10 patients suffering from dry eye disease. Corneal permeability measurements were performed by fluorophotometry after instilling $40 \mu \mathrm{L}$ of a solution of $2 \%$ sodium fluorescein, before and after treatment with the HP-Guar drops. The results were analysed using Wilcoxon test for paired data.

Results: A mean decrease in corneal permeability of $45 \%$, after the use of HP-Guar drops was found $(\mathrm{p}=0.002)$.
\end{abstract}

Recibido: 15/2/06. Aceptado: 14/6/06.

Hospital Universitario Clínico San Carlos. Universidad Complutense. Madrid. España.

1 Licenciado en Medicina.

2 Doctor en Medicina.

Los autores declaran no tener interés comercial en el HP-Guar o cualquiera de los productos, técnicas o equipos empleados para la realización del trabajo, de conformidad con la normativa editorial de Archivos.

Correspondencia:

Ignacio Cerván López

Hospital Universitario Clínico San Carlos

Pabellón 8, 5. ${ }^{\text {a }}$ planta

28003 Madrid

España

E-mail: icervan@yahoo.com 
Resultados: Se observó tuvo una disminución estadísticamente significativa de la permeabilidad corneal media del $45 \%$ tras tratamiento con HP-Guar $(\mathrm{p}=0,002)$.

Conclusiones: El HP-Guar constituye una nueva opción terapéutica que ha demostrado fluotofotométricamente reducir la permeabilidad epitelial corneal tras su uso.

Palabras clave: Fluorofotometría, HP-Guar, ojo seco, permeabilidad epitelial, lagrimas artificiales.
Conclusions: HP-Guar provides a new therapeutic option significantly decreasing corneal epithelial permeability (Arch Soc Esp Oftalmol 2006; 81: 327-332).

Key words: Fluorophotometry, HP-Guar, dry eye, epithelial permeability, artificial tears.

\section{INTRODUCCIÓN}

El ojo seco o queratoconjuntivitis sicca se define como «un desorden de la película lagrimal debido a una deficiencia lagrimal o a un exceso de evaporación que causa alteraciones en la superficie ocular interpalpebral y se asocia con síntomas de disconfort» (1). Su prevalencia en la población adulta se estima entre $10-20 \%$ (2).

La córnea cumple varias funciones: refractiva, superficie de intercambio de $\mathrm{O}_{2}$ y nutrientes, ayuda al sostén de la película lagrimal. Además de estas funciones hay que añadir otra: constituye la primera barrera física del globo ocular. A esto contribuye en gran medida su capa más externa, un epitelio no queratinizado, estratificado y escamoso. Sólo el epitelio corneal representa más del $50 \%$ de la función de barrera de una córnea sana. La unión de estas células son de tipo «zonula occludens» en su extremo apical, desmosomas o hemidesmosomas en el resto. Dichas uniones hacen que una sustancia liposoluble tenga pocas o ninguna posibilidad de acceder al estroma, y bloquea la entrada a sustancias polarizadas como el agua o iones. Cualquier causa que altere estas uniones supondría la pérdida de esta función de barrera.

La película lagrimal es una capa muy especializada y bien organizada que cubre, entre otras áreas, el epitelio corneal. Alteraciones de la película lagrimal que afecten a su composición o volumen pueden resultar a corto plazo en una seria alteración de la superficie ocular. La superficie ocular debe considerarse como una unidad funcional compuesta por la conjuntiva, córnea, párpados, glándulas lagrimales, e inervación de todo ese conjunto. Una enfermedad o disfunción de alguna parte de esa unidad funcional desemboca en una película lagrimal inestable (3-6). Esto se traduce en un daño funcional epitelial afectando a las uniones celulares, lo que conduce a una rotura de la impermeabilidad de la barrera epitelial, observándose en casos avanzados una queratopatía: queratoconjuntivitis sicca.

La fluorofotometría es la única técnica objetiva para valorar in vivo la función de la barrera epitelial, empleando como trazador la fluoresceína (7). Hasta mínimas lesiones de la superficie corneal, no detectables con una exploración oftalmológica con lámpara de hendidura pueden provocar una rotura de la barrera epitelial cuantificable sólo por fluorofotometría (7-11).

El tratamiento habitual del ojo seco son los sustitutos lagrimales que humidifican la superficie ocular. El HP Guar (hidroxipropil guar) es un compuesto novedoso en el tratamiento del ojo seco, cuyas propiedades $(12,13)$ no sólo permiten la humidificación sino también la lubricación y reparación de las lesiones epiteliales.

El objetivo de este estudio es valorar fluorofotométricamente el papel del HP-Guar en el tratamiento de los pacientes con ojos seco.

\section{SUJETOS, MATERIAL Y MÉTODO}

Se realizó un estudio prospectivo, enmascarado, pareado, sobre 10 pacientes con ojos secos, siendo examinados 10 ojos (un ojo por paciente). Para evitar la selección de dos ojos por sujeto se determinó estudiar en todos los casos el ojo izquierdo tras sorteo en el primer paciente. Todos los pacientes fueron mujeres con una edad media de 60,3 años (DE: 3,4 años).

Los criterios de inclusión fueron: test de Schirmer tipo 1 menor de $10 \mathrm{~mm}$ (14) y sintomatología de ojo seco: sensación de cuerpo extraño, sensación de «pinchazos», escozor o visión borrosa intermitente. 
Los criterios de exclusión fueron: ser portador de lentes de contacto, cirugías oftalmológicas previas, o enfermedad sistémica que pueda alterar la superficie ocular.

Los pacientes dejaron su tratamiento previo con lágrimas artificiales en ese ojo una semana antes del estudio.

A todos los pacientes se le realizó un estudio fluorofotométrico antes y después del tratamiento con HP-Guar. Se ha utilizado para el análisis el fluorofotómetro Fluorotron Master (Ocumetrics, California, EEUU).

En cada una de esas visitas se le tomaron primero tres medidas de la autofluorescencia corneal, realizando la media de dichas medidas; a continuación se instilaba 40 microlitros de una solución estéril de fluoresceína sódica al $2 \%$ en fondo de saco, y se esperaron 45 minutos antes de realizar las siguientes tres mediciones, y su correspondiente media. Una vez finalizado el estudio pretratamiento se pautó colirio de HP-Guar (Systane ${ }^{\circledR}$, Alcon, España), 4 instilaciones diarias durante un mes. La permeabilidad corneal (P) o estado de la barrera epitelial fue de [P45']-[P0'], donde $\mathrm{P}^{\prime} 5^{\prime}$ es el valor medio de fluorescencia estromal transcurridos 45 minutos después de la instilación de fluoresceína y $\mathrm{PO}^{\prime}$ la fluorescencia estromal o autofluorescencia antes de la misma $(10,11)$.

Antes y después del tratamiento los pacientes fueron explorados en lámpara de hendidura. Se los clasificó del 1 al 3 según la triple Clasificación de Madrid para el ojo seco (15): 1= leve (síntomas sin signos); $2=$ moderado (síntomas con signos reversibles); $3=$ (síntomas con signos irreversibles).

Se consideró significativa una $\mathrm{p}<0,05$. Se analizaron los resultados aplicando un test de Wilcoxon para datos pareados.

\section{RESULTADOS}

Los valores se agruparon en 2 categorías: pre HPGuar y en post HP-Guar. Los resultados se expresan en la tabla I.

La media de los valores de P pre HP-Guar con un intervalo de confianza (IC) del 95\% fue de 1.228,18 $\mathrm{ng} / \mathrm{ml}$ y postratamiento fue $629,81 \mathrm{ng} / \mathrm{ml}$. La mediana pre HP-Guar (IC del 95\%) fue de 747,60 $\mathrm{ng} / \mathrm{ml}$, y postratamiento fue $332,95 \mathrm{ng} / \mathrm{ml}$. La desviación estándar pretratamiento fue de 1.244,23 $\mathrm{ng} / \mathrm{ml}$, y postratamiento fue de $757,85 \mathrm{ng} / \mathrm{ml}$.

Como se puede observar en la figura 1 cuanto mayor era la permeabilidad pre HP-Guar, mayor era la reducción de la misma con el tratamiento. El porcentaje medio de descenso de la permeabilidad fue de un $45,96 \%$. Se obtuvo una diferencia estadísticamente significativa $\mathrm{p}=0,002$, existiendo una mejoría de la función de barrera tras el tratamiento.

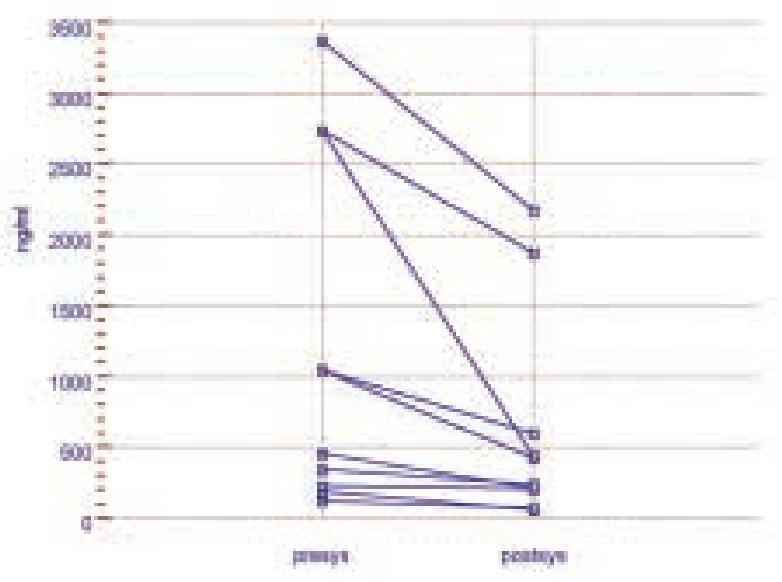

Fig. 1: Disminución de la permeabilidad epitelial antes $y$ tras el tratamiento, en cada paciente.

Tabla I. Resultados de la permeabilidad (P) media fluororofotométrica de la muestra

\begin{tabular}{lccc}
\hline & P Pre HP-Guar $(\mathrm{ng} / \mathrm{ml})$ & P Post HP-Guar $(\mathrm{ng} / \mathrm{ml})$ & Reducción permeabilidad en $\%$ \\
\hline Paciente 1 & 452,7 & 208,9 & 53,85 \\
Paciente 2 & 2743,2 & 1873,2 & 31,71 \\
Paciente 3 & 190,1 & 57,6 & 69,70 \\
Paciente 4 & 1048,3 & 425,9 & 59,37 \\
Paciente 5 & 233,1 & 201,3 & 13,64 \\
Paciente 6 & 1042,5 & 602,1 & 42,24 \\
Paciente 7 & 3375,2 & 2176,8 & 35,50 \\
Paciente 8 & 119,8 & 71,5 & 40,32 \\
Paciente 9 & 340,3 & 240 & 29,47 \\
Paciente 10 & 2736,6 & 440,8 & 83,89 \\
\hline \hline
\end{tabular}

P: permeabilidad. 
Previo al tratamiento se clasificaron a los pacientes como ojo seco moderado (7 pacientes) y leve (3 pacientes). De los ojos moderados, 4 presentaban tinciones corneales punteadas de más de la mitad del epitelio corneal, y los restantes 3 sólo alteraciones mínimas a nivel inferior. Tras el tratamiento 7 de los 10 pacientes afirmaron notar clara mejoría subjetiva de los síntomas de sensación de cuerpo extraño, de dolor en "pinchazos», escozor, y la visión borrosa intermitente, sin desaparecer por completo ninguno de ellos. Todos los pacientes disminuyeron su grado de tinción corneal salvo uno, aunque sí refirió mejoría sintomática. En resumen, se apreció una mejoría subjetiva clara en todos los parámetros sintomáticos analizados, y mejoría leve de la tinción corneal en general.

\section{DISCUSIÓN}

La barrera epitelial corneal constituye la más importante a nivel corneal. Su integridad depende, entre otros factores, de una buena lubricación lagrimal. Sin esto, un epitelio sano, tiende a descamarse y a erosionarse.

Existen varias técnicas para el estudio del epitelio corneal: microscopía especular, microscopia confocal, microscopía electrónica, cultivos celulares, medidas electrofisiológicas, imágenes de Scheimpflug, RMN protónica y la fluorofotometría.

Si la exploración corneal revela queratopatía seca, esta queratopatía también se vera reflejada en el análisis fluorofotométrico (16); pero si la exploración clínica es normal, puede existir una alteración de la barrera epitelial subclínica sólo valorable con la fluorofotometría. Esto es debido a que la fluorofotometría es la única técnica objetiva para valorar la función barrera del epitelio corneal. La fluorofotometría ha sido empleada para el estudio de la permeabilidad del epitelio en múltiples circunstancias: autofluorescencia $(17,18)$, en usuarios de lentes de contacto (1921 ), en tratamientos tópicos con y sin conservantes (22-29), después de un tratamiento quirúrgico, en pacientes con ojo seco de diversa etiología $(30,31)$, en patologías sistémicas (32-35) y para estudios de fisiología corneal (36-41). En un trabajo nuestro anterior observamos que el valor de permeabilidad epitelial corneal en sujetos normales es $34,4 \pm 23,5$ ng/ml (27). Así vemos cómo los pacientes de este estudio con ojo seco tienen una permeabilidad mucho más elevada que los normales.
Se han realizado estudios fluorofotométricos sobre otros tipos de lágrimas artificiales (37), y sus conservantes, y éste es el primero que se realiza sobre el HP-Guar. El alcohol polivinílico ha demostrado su capacidad para disminuir la permeabilidad hasta en un $44 \%$, similar a nuestros resultados, posiblemente por su acción mucomimética $(38,39)$.

El HP-Guar (Systane, Alcon, España) es un principio activo nuevo que ha demostrado disminuir los síntomas y signos de la sequedad ocular a lo largo del día en comparación con la carboximetilcelulosa (disminuía la sensación de cuerpo extraño y mejoraba la sensación de sequedad). El HP-Guar presenta actividad mucomimética, protege el epitelio corneal a largo plazo y favorece además su función de barrera al facilitar la regeneración epitelial $(9,40)$. Este principio esta acompañado de los demulcentes polietilenglicol 400 (PEG 400) y propilenglicol, y hay que considerar el conjunto de los 3 como el «principio activo» del producto.

Los conservantes son sustancias que protegen los preparados oftalmológicos contra la contaminación y el crecimiento de microorganismos patógenos. Los conservantes utilizados actualmente actúan contra la membrana celular bacteriana, lesionándola o destruyéndola. Todos los conservantes, con suficiente eficacia contra los microorganismos, presentan efectos tóxicos y reacciones alérgicas variables. Los pacientes que usan colirios con frecuencia, como lo son los pacientes con ojos seco, son los más expuestos a estos efectos adversos. La gran capacidad de almacenamiento de la córnea y la conjuntiva facilita las reacciones tóxicas de los conservantes. Incluso a bajas concentraciones estas sustancias disminuyen considerablemente la resistencia epitelial de la córnea. Los conservantes más utilizados son el cloruro de benzalconio, clorhexidina, la cetrimida, el clorobutanol, el tiomerosal, el cloruro amónico policuaternario (polyquad) y las biguanidas. De todos ellos el más tóxico parece ser el cloruro de benzalconio (41-43). El conservante principal del HPGuar es el polyquad. En un estudio in vitro sobre cultivo de células epiteliales corneales humanas realizado por Tripathi et al el polyquad mostró no tener efectos distinguibles sobre la actividad mitótica ni sobre los movimientos citoquinéticos de las células epiteliales (43). De modo que aunque la formulación de HP-Guar lleva conservante su efecto mucomimético (9) contrarresta la escasa toxicidad del polyquad. 
Éste es el primer estudio que valora el efecto del HP-Guar sobre la función de la barrera epitelial, medido con fluorofotometría. Los rangos amplios de valores de la media y la mediana de nuestros resultados se explican por presentar nuestra muestra pacientes con distintos grados de lesión epitelial punteada previa. Los resultados, tanto en la reducción de la permeabilidad, como en su disminución de la sintomatología, nos lleva a valorar el HP-Guar como un tratamiento eficaz y sin toxicidad para el epitelio corneal. Nuestro estudio aún presentando una muestra pequeña, es prometedor; y sería interesante realizar otro estudio con una muestra mayor y valorar también los resultados del HP-Guar sin conservantes en pacientes con distintos grados de sequedad ocular .

En conclusión, el tratamiento con HP-Guar mejora objetivamente la rotura de la barrera epitelial. Por otra parte reduce los síntomas de sequedad ocular.

\section{BIBLIOGRAFÍA}

1. Lemp MA. Report of the National Eye Institute/Industry workshop on Clinical Trials in Dry Eyes. CLAO J 1995; 21: 221-232.

2. Mather WD. Why the eye becomes dry: a cornea and lacrimal gland feedback model. CLAO J 2000; 26: 159165.

3. Mircheff AK. Hormonal support of lacrimal function, primary lacrimal deficiency, autoinmunity and peripheral tolerance in the lacrimal gland. Ocul Immunol Inflamm 1996; 4: 145-152.

4. Azzarolo AM, Mircheff AK, Kaswan RL, Stanczyk FZ, Gentschein E, Becker L. Androgen support of lacrimal gland function. Endocrine 1997; 6: 39-45.

5. Stern ME, Beuerman RW, Fox RI, Gao J, Mircheff AK, Pflugfelder SC. The pathology of dry eye: the interaction between the ocular surface and lacrimal glands. Cornea 1998; 17: 584-589.

6. Jones DT, Monroy D, Ji Z, Pflugfelder SC. Alterations of ocular surface gene expresión in Sjogren's syndrome. Adv Exp Med Biol 1998; 438: 533-536.

7. de Kruijf EJ, Boot JP, Laterveer L, van Best JA, Ramselaar JA, Oosterhuis JA. A simple method for determination of corneal epithelial permeability in humans. Curr Eye Res 1987; 6: 1327-1334.

8. Araie M, Maurice D. The rate of diffusion of fluorophores through the corneal epithelium and stroma. Exp Eye Res 1987; 44: 73-87.

9. Gobbels M, Spitznas M. Corneal epithelial permeability of dry eyes before and after treatment with artificial tears. Ophthalmology 1992; 99: 873-878.

10. Gobbels M, Spitznas M. Effects of artificial tears on corneal epithelial permeability in dry eyes. Graefes Arch Clin Exp Ophthalmol 1991; 229: 345-349.
11. Gobbels M, Spitznas M. Influence of artificial tears on corneal epithelim in dry-eye syndrome. Graefes Arch Clin Exp Ophthalmol 1989; 227: 139-141.

12. Ubels JL, Clousing DP, Van Haitsma TA, Hong BS, Stauffer $P$, Asgharian B, et al. Pre-clinical investigation of the efficacy of an artificial tear solution containing hydroxypropyl-guar as a gelling agent. Curr Eye Res 2004; 28. 437-444.

13. Hartstein I, Khwarg S, Przydryga J. An open-label evaluation of HP-Guar gellable lubricant eye drops for the improvement of dry eye signs and symtoms in a moderate dry eye adult population. Curr Med Res Opin 2005; 21: 255-260.

14. Clinch TE, Benedetto DA, Felberg NT, Laibson PR. Schirmer's test. A closer look. Arch Ophthalmol 1983; 101 . 1383-1386.

15. Murube J, Benítez del Castillo JM, Chenzhuo L, Berta A, Rolando M. The Madrid triple classification of dry eye. Arch Soc Esp Oftalmol 2003; 78: 587-593.

16. Miyata K, Amano S, Sawa M, Nishida T. A novel grading method for superficial punctate keratopathy magnitude and its correlation with corneal epithelial permeability. Arch Ophthalmol 2003; 121: 1537-1539.

17. Yokoi N, Kinoshita S, Akiyama K. A new slit-lamp fluorophotometer for the clinical evaluation of corneal epithelial barrier function. Nippon Ganka Gakkai Zasshi 1994; 98: 641-647.

18. Boets EP, van Best JA. Corneal autofluorescence and epithelial barrier function. Cornea 1994; 13: 373-374.

19. Ichijima H, Yokoi N, Nishizawa A, Kinoshita S. Fluorophotometric assessment of rabbit corneal epithelial barrier function after rigid contact lens wear. Cornea 1999; 18. 87-91.

20. McNamara NA, Polse KA, Fukunaga SA, Maebori JS, Suzuki RM. Soft lens extended wear affects epithelial barrier function. Ophthalmology 1998; 105: 2330-2335.

21. Boets EP, van Best JA, Boot JP, Oosterhuis JA. Corneal epithelial permeability and daily contact lens wear as determined by fluorophotometry. Curr Eye Res 1988; 7: 511-514.

22. Niiya A, Yokoi N, Matsumoto Y, Komuro A, Ishibashi T, Tomii S, et al. Effect of beta-blocker eyedrops on corneal epithelial barrier function. Ophthalmologica 2000; 214. 332-336.

23. McCarey BE, Reaves TA. Effects of tear lubricating solutions on in vivo corneal epithelial permeability. Curr Eye Res 1997; 16: 44-50.

24. Shimazaki J, Fujishima H, Yagi Y, Tsubota K. Effects of diclofenac eye drops on corneal epithelium structure and function after small-incision cataract surgery. Ophthalmology 1996; 103: 50-57.

25. Benitez del Castillo JM, Castillo A, Toledano N, Duran S, del Aguila $C$, Otero $M$, et al. Influence of topical cyclosporine A and dissolvent on corneal epithelium permeability of fluorescein. Doc Ophthalmol 1995; 91: 49-55.

26. de Jong C, Stolwijk T, Kuppens E, de Keizer R, van Best J. Topical timolol with and without benzalkonium chloride: epithelial permeability and autofluorescence of the cornea in glaucoma. Graefes Arch Clin Exp Ophthalmol 1994; 232: 221-224.

27. Benitez del Castillo JM, del Aguila C, Duran S, Hernandez. J, Garcia Sánchez J. Influence of topically applied 
cyclosporine A in olive oil on corneal epithelium permeability. Cornea 1994; 13: 136-140.

28. Gobbels M, Spitznas M. Corneal epithelial permeability of dry eyes before and after treatment with artificial tears. Ophthalmology 1992; 99: 873-878.

29. Ramselaar JA, Boot JP, van Haeringen NJ, van Best JA, Oosterhuis JA. Corneal epithelial permeability after instillation of ophthalmic solutions containing local anaesthetics and preservatives. Curr Eye Res 1988; 7: 947-950.

30. Dursun D, Wang M, Monroy D, Li DQ, Lokeshwar BL, Stern ME, et al. A mouse model of keratoconjunctivitis sicca. Invest Ophthalmol Vis Sci 2002; 43: 632-638.

31. Yokoi N, Kinoshita S. Clinical evaluation of corneal epithelial barrier function with the slit-lamp fluorophotometer. Cornea 1995; 14: 485-489.

32. Khalil HA, van Best JA, de Keizer RJ. The permeability of the corneal epithelium of Graves' ophthalmopathy as determined by fluorophotometry. Doc Ophthalmol 1989; 73: 249-254.

33. Stolwijk TR, van Best JA, Boor JP, Lemkes HH, Oosterhuis JA. Corneal epithelial barrier function after oxybuprocaine provocation in diabetics. Invest Ophthalmol Vis Sci 1990; 31: 436-439.

34. Chang SW, Hsu HC, Chen MS. Corneal autofluorescence and epithelial barrier function in diabetic patients. Ophthalmic Res 1995; 27: 74-79.

35. Yokoi K, Yokoi N, Kinoshita S. Impairment of ocular surface epithelium barrier function in patients with atopic dermatitis. Br J Ophthalmol 1998; 82: 797-800.
36. Nakamura M, Kawahara M, Nakata K, Nishida T. Restoration of corneal epithelial barrier function and wound healing by substance $P$ and IGF-1 in rats with capsaicininduced neurotrophic keratopathy. Invest Ophthalmol Vis Sci 2003; 44: 2937-2940.

37. Goebbels M, Spitznas M. Effects of artificial tears on corneal epithelial permeability in dry eyes. Graefes Arch Clin Exp Ophthalmol 1991; 229: 345-349.

38. Norn MS, Opauszki A. Effects of ophthalmic vehicles on the stability of the precorneal tear film. Acta Ophthalmol (Copenh) 1977; 55: 23-34.

39. Krishna N, Brow F. Polyvinyl alcohol as an ophthalmic vehicle. Effect on regeneration of corneal epithelium. Am J Ophthalmol 1964; 57: 99-106.

40. Christensen MT, Cohen S, Rinelhart J, Akers F, Pemberton B, Bloomenstein $M$, et al. Clinical evaluation of an HP-guar gellable lubricant eye drop for the relief of dryness of the eye. Curr Eye Res 2004; 28: 55-62.

41. Lopez Bernal D, Ubels JL. Artificial tear composition and promotion of recovery of the damaged corneal epithelium. Cornea 1993; 12: 115-120.

42. Lopez Bernal D, Ubels JL. Quantitative evaluation of the corneal epithelial barrier: effect of artificial tears and preservatives. Curr Eye Res 1991; 10: 645-656.

43. Tripathi BJ, Tripati RC, Kolli SP. Cytotoxicity of ophthalmic preservatives on human corneal epithelium. Lens Eye Toxic Res 1992; 9: 361-367. 